Gut, 1960, 1, 76.

\title{
VOLVULUS OF THE SMALL INTESTINE IN ADULTS
}

\author{
BY
}

\author{
C. H. TALBOT
}

From the United Bristol Hospitals

Five cases of volvulus of varying lengths of the small intestine are described. The incidence and the aetiology of the condition are briefly discussed.

Shock as a feature of extensive volvulus is stressed, and its cause in these cases is related to the previous animal experimental work of others. The other clinical features are briefly described.

In the management of these cases the urgency for laparotomy is stressed and immediate delivery from the abdomen of the whole small bowel is advocated. Reference is made to the literature of massive resection of the small intestine to illustrate that the prognosis is not necessarily poor when resections are extensive.

Acute volvulus of the small intestine is one of the most serious abdominal emergencies. Diagnosis may be difficult and delay in operative intervention can be disastrous. When the whole or a major portion of the small bowel mesentery is twisted the patient may deteriorate with alarming rapidity.

The main purpose of this paper is to show how the clinical picture may be expected to vary and to emphasize the importance of "shock" in massive volvulus. Not only is it of interest to correlate the shock seen in some of these cases with the experimental work of others (Aird, 1937; Scott, 1938) but an understanding of the factors involved should lead to rational treatment.

\section{CASE RePorts}

CASE 1.-A man, aged 47, who had undergone a Polya gastrectomy three years previously for duodenal ulcer was admitted on October 14, 1957, complaining of colicky upper abdominal pain of eight hours' duration. Four hours before admission he passed a normal motion and then felt faint. He took a hot drink and then vomited several times. On admission when seen by the house surgeon he was pale, cold and clammy, with a poor pulse volume and a blood pressure of $90 / 60 \mathrm{~mm}$. $\mathrm{Hg}$. Twenty minutes later when I saw him he had a warm periphery, no pallor, and a blood pressure of $120 / 80 \mathrm{~mm}$. $\mathrm{Hg}$. There was tenderness in the upper abdomen and a suggestion of central abdominal distension. A straight radiograph of the abdomen showed a few dilated small bowel loops with fluid levels. Another period of shock was then observed and an intravenous saline infusion was started in preparation for laparotomy.

Under general anaesthesia the abdomen was explored through an upper left paramedian incision. A volvulus of the whole small bowel was found, the mesentery being twisted anticlockwise about $180^{\circ}$. The twist was quite loose, causing only slight venous congestion in the mesentery. The small intestine was moderately distended throughout its length. The previous gastrectomy appeared in good order and there were no adhesions to the small intestine. After delivering the small bowel from the abdomen the only apparent abnormality was that the root of the mesentery had a rather short attachment to the posterior abdominal wall. An attempt to lengthen this attachment was made by suturing the terminal ileum below the caecum to the posterior parietal peritoneum and stitching the upper jejunal mesentery to the posterior abdominal wall medial to the descending colon. The post-operative course was uneventful and the patient was well three months later.

CASE 2.-A woman of 50 was admitted on December 29, 1957, with a history of severe central abdominal pain radiating into the back for 16 hours. The pain was intermittent and colicky at first but became continuous. She had vomited a small amount of green fluid three times. She had a bowel action soon after the onset of the pain but had not passed faeces or flatus since.

She said that for two months before admission her abdomen had been swelling periodically. She would sometimes bend forward and feel her stomach "roll over". This would be followed by distension and heaviness and was relieved by the passing of flatus.

On examination she was pale and the periphery was cold but not blue. Her blood pressure was $90 / 60 \mathrm{~mm}$. $\mathrm{Hg}$. The lower abdomen was distended, and a soft mass; which was dull to percussion, appeared to rise out of the pelvis and to reach a point just above the umbilicus. Although the whole abdomen was tender the maximal tenderness occurred over the lower abdominal mass; no peristaltic sounds were heard. It was at first thought that this was a case of twisted ovarian tumour probably with rupture and haemorrhage. When the haemoglobin was reported as $130^{\circ}$, reconsideration of the clinical 
picture led to a diagnosis of strangulating small bowel obstruction.

The stomach was emptied with a gastric tube and an intravenous saline infusion started. The abdomen was explored under general anaesthesia through a right paramedian incision within an hour of admission. As the incision was being made the anaesthetist reported serious deterioration in the patient whose pulse was barely palpable. A second intravenous drip was then started through which blood was given. On opening the abdomen the whole small bowel was seen to be distended and congested. It was all immediately delivered from the abdomen and a volvulus of the whole small bowel on its mesentery was seen. The colour changes in the distal half of the small gut were fairly advanced, and it was with considerable relief that the colour of the bowel was seen to improve on untwisting the mesentery. A small linear area of doubtful viability where the terminal ileum had been stretched over the twisted mesentery was buried with a Lembert suture. The small intestine was returned to the abdomen all clearly viable. By the time the incision was closed the patient's condition was good, the pulse was of good volume, and the blood pressure almost normal. By the end of the operation the patient had received 1 litre of saline and $300 \mathrm{ml}$. of blood.

Intravenous therapy and gastric suction were continued for 72 hours. Thereafter, apart from slight abdominal distension which took a week to subside, convalescence was uneventful.

CASE 3.-A man aged 38 was admitted as an emergency on August 30, 1958. For some years he had passed frequent loose stools. He had been investigated elsewhere and labelled as a case of mild colitis.

Forty-eight hours before admission he developed epigastric pain. After a night's rest he felt well enough to go to work but during the day the pain returned and grew more severe. During the 24 hours before admission he had severe, piercing epigastric pain radiating through to the back. The pain was made worse by movement. He vomited some green fluid just before admission and about half a litre of bright red blood after admission. Some hours earlier he had passed some loose stools and flatus.

On examination he was pale with a cold periphery and a pulse of poor volume. His blood pressure was $90 / 60 \mathrm{~mm}$. Hg. The abdomen was tender and rigid. The upper abdomen was most rigid. No peristaltic sounds were heard. During the next two hours he received 1 litre of plasma, $1 \frac{1}{2}$ litres of blood, and 1 litre of saline. His general condition was then sufficiently improved for laparotomy.

The abdomen was explored under general anaesthesia through an upper median incision. A volvulus of the jejunum was found without any apparent causative factor. The oedematous mesentery was tightly twisted clockwise, and $7 \mathrm{ft}$. of bowel was devitalized with a sharp line of demarcation at each end. The gangrenous gut was resected and an end-to-end anastomosis of bowel with a vigorous blood supply performed 4 in. below the duodeno-jejunal flexure. One further litre of blood and half a litre of plasma were given during the operation.
Post-operatively the patient progressed satisfactorily until the third post-operative day when his pulse began to rise and he rapidly became shocked. The abdomen was found to be tender with some rigidity and rebound tenderness on the left side.

A litre of plasma was given and the abdomen was again explored under general anaesthesia. A further $4 \mathrm{ft}$. of small bowel immediately distal to the anastomosis was found to be gangrenous, due to thrombosis in the small peripheral mesenteric veins. Again resection and end-toend anastomosis were performed, leaving about $4 \mathrm{ft}$. of terminal ileum and 3 in. of proximal jejunum. He received half a litre each of blood and plasma during operation and his immediate post-operative condition was quite good. In view of the mesenteric thrombosis found at the second operation, heparin, 12,000 units six hourly, was given intravenously for four days. By the fourth day after the second operation he had developed extensive collapse of the left lower lobe with areas of consolidation. The clinical course was stormy for 10 days with high fever and mental confusion. The infection slowly resolved with the assistance of physiotherapy, erythromycin, and chloramphenicol. The patient's alimentary canal gave remarkably little trouble, and at the time of his discharge four weeks after admission he was taking a normal diet and passing two or three loose stools a day. Three months later he had gained a stone in weight, he was having two bowel actions a day, and examination of the stool showed no steatorrhoea. The blood electrolytes were normal. He remains well six months later.

CASE 4.-A man of 67 was admitted on April 30, 1958, with a 12-hour history of colicky central abdominal pain of sudden onset. He had not vomited but he could not recollect passing flatus since the onset of the pain.

For the past 30 years he had suffered slight epigastric pain related to food and relieved by alkalis.

He was a fit man in good general condition. There was very slight central abdominal distension but no localized or rebound tenderness. A freely reducible right inguinal hernia appeared to be an incidental finding.

An hour after admission the abdomen was explored through a right paramedian incision under general anaesthesia. The small bowel was moderately distended and some straw-coloured free fluid was found. A volvulus of a short loop of ileum had occurred, at the apex of which lay a large multilobular subserous lipoma. The volvulus was not a tight one and although the lumen had been obstructed by it the blood supply of the loop was not yet affected. Ten centimetres of ileum bearing the lipoma were resected and an end-to-end anastomosis performed.

The post-operative course was smooth.

CASE 5.-A man of 48 was admitted on March 19, 1958, with a history of constant upper abdominal pain with colicky exacerbations lasting three hours. He had vomited green fluid three times. Eight years previously he had undergone a Billroth I partial gastrectomy for duodenal ulcer.

He was a well-built man whose general condition was 
good. He was rolling about with spasms of pain. There was slight upper abdominal distension and generalized abdominal tenderness with guarding in the upper abdomen. Peristaltic sounds were markedly increased.

Acute upper small bowel obstruction was disgnosed and immediate laparotomy arranged. The abdomen was opened under general anaesthesis through the old scar. Four to five feet of upper jejunum was distended and the mesentery of the distended segment was twisted. The apex of the twisted loop was adherent to the old wound. The adhesion was divided and the loop untwisted; its blood supply was not impaired. A few small bowel adhesions were severed and the abdomen closed.

He developed a silent abdominal distension postoperatively and he did not pass flatus for four days. Thereafter convalescence was smooth.

\section{Discussion}

INCIDENCE.-No one person is likely to see very many of these cases and unfamiliarity with a variable clinical picture probably still contributes to a considerable mortality. McKechnie and Priestley (1936) found only 37 cases in reviewing the condition over 25 years at the Mayo Clinic, and Moretz and Morton (1950) found 36 cases in 22 years at the University of Rochester.

Aetiology.-In most published series of volvulus of the small bowel the majority of cases show some definite aetiological factor. Congenital malrotation and congenital or post-operative adhesions are the commonest causes. Tumours, as in Case 4, are occasionally responsible and primary mesenteric vascular occlusion is sometimes blamed. In Cases 1 and 2, in which the volvulus was total, and in Case 3, in which the volvulus was subtotal, no definite predisposing cause was found. Case 1 appeared to have a mesentery which was long in relation to the length of attachment of its root. Kerr and KirkaldyWillis (1946) describe seven cases of total small bowel volvulus in East Africans in whom volvulus is a relatively common cause of obstruction of the small bowel. They suggested that the racial dietary habit of taking large quantities of maize gruel at one time might load the small intestine and initiate volvulus. The small bowel might become loaded very quickly more easily in a patient, such as Case 1, who had been subjected to a gastrectomy. The history of this case shows nothing to support this supposition.

Cases 2 and 3 were too critically ill to justify a prolonged search for possible causative factors.

SHock.-In cases where the volvulus involves the whole or a large part of the small intestine, "shock", as in Cases 1,2, and 3, is a prominent feature. It is not necessarily a late sign. When the whole or a substantial portion of the small bowel mesentery becomes twisted the venous return soon becomes obstructed. Aird (1937) showed experimentally that the maximum loss of whole blood in intestinal strangulation occurred with pure venous occlusion. He showed that $50 \%$ of the blood volume could be lost into the splanchnic bed. The episodes of hypotension, pallor, and sweating in Case 1 are adequately explained if the twist of the whole mesentery was a slight one, the veins becoming temporarily obstructed and relieved by spontaneous partial untwisting of the volvulus. The operative finding of a loosely twisted mesentery, minimal congestion of the gut, and minimal peritoneal exudation supports this explanation.

Scott (1938) made a detailed study of the shockproducing factor in experimentally produced intestinal strangulation in dogs. He found that, if the venous return alone were occluded in a closed loop obstruction, death occurred earlier than in pure arterial or complete arterial and venous occlusion. In pure venous occlusion $60 \%$ of the animal's blood volume was lost into the lumen, bowel wall, and coelomic cavity. In pure arterial occlusion transudation of plasma into the peritoneal cavity as a response to the presence of a necrotic segment of bowel was the most important shock-producing factor. It is to be expected, therefore, that some cases of volvulus of the small bowel will become shocked first from blood loss as a result of venous occlusion and later from protein and fluid exudation. This combination is illustrated in Case 2. The patient's very rapid recovery during the short operation can be explained by the return to the general circulation of a large volume of blood in the congested mesenteric veins on untwisting the volvulus. The small amount of fluid and blood given intravenously during operation could hardly explain such a dramatic and rapid recovery. The twice-checked haemoglobin of $120 \%$ in this case illustrates that a great amount of protein and fluid as well as blood is lost.

Scott also showed that absorption of bacterial toxins from the peritoneal cavity as a shockproducing factor was a late phenomenon occurring after the gut wall had become necrotic. Even in Case 3 this stage had not been reached, since the shock state was reversed as soon as the estimated blood and fluid loss had been replaced.

PaIN.-This is usually severe and gripping in character but often continuous. Pain is usually the dominant symptom in small loop volvulus and, as in Case 4, it may be the only definite clinical feature.

VomITING.-Vomiting may be absent or slight in a case which urgently needs laparotomy. Evans and Bigger (1947) say that vomiting is usually incessant 
in strangulating obstruction but Kerr and KirkaldyWillis (1946) describe six cases of small-bowel volvulus in which vomiting was not a marked feature. It is clear that incessant vomiting is not necessarily a symptom. Blood may be vomited, as in Case 3, when venous obstruction in the mesentery precedes occlusion of the lumen of the proximal limb of the volvulus.

Bowels.-The patient, as in Case 2, may have one or more bowel actions after the onset of the pain. Blood may be passed if the lumen of the distal limb of the volvulus is incompletely occluded. Usually complete constipation to faeces and flatus follows; its significance may not be great if the history is short.

Abdominal Physical Signs.-Obvious distension was present in Case 2 only. The diagnosis must often be made in its absence. Tenderness may be slight when there is minimal congestion of the bowel (Case 1), or gross and accompanied by rigidity, as in Case 3, when bowel is devitalized. Peristaltic sounds may be absent (Cases 2 and 3), diminished (Case 1), normal (Case 4), or increased (Case 5). Smith (1945) suggests that colic associated with absent bowel sounds is typical of strangulation, and Evans and Bigger (1947) say that the sounds are diminished in frequency and lower in pitch in strangulating obstruction. The behaviour of peristaltic sounds appears to vary with the amount of mesentery twisted and the degree of vascular impairment of the bowel.

MANAGEMENT.-The exact diagnosis may not be made pre-operatively but it is usually possible to decide that a serious abdominal disaster has occurred. Moretz and Morton (1950) say that the chief problem is differentiation between an obstruction needing surgery and one which can be treated conservatively. This problem disappears when it is accepted that, with the occasional exception of the much-scarred abdomen, all cases of acute small intestinal obstruction require laparotomy (Cooke, 1958) since it is impossible to differentiate certainly between occlusion and strangulation.

In cases of massive volvulus the circulatory volume must be restored with blood and plasma pre-operatively. Much is to be gained by the earliest possible operative intervention; in Case $2 \mathrm{a}$ resection of gut of some magnitude might have been necessary if too complete a resuscitation had been attempted, although perhaps we erred on the side of haste in opening this abdomen. Provided the patient is made fit for general anaesthesia further resuscitation and operation can be synchronous. Much unnecessary delay can be avoided in the operative diagnosis by immediate delivery of the whole small gut from the abdomen (Barbosa, 1944). In extensive volvulus, it is in any case necessary to deliver the small intestine into the abdomen in order to untwist the mesentery. When there are many dilated small bowel loops, attempts at finding the obstructing lesion while keeping the bowel in the abdomen are fruitless, and volvulus may even be completely overlooked (Woods, 1945).

There is no alternative to massive resection when the bowel is gangrenous as in Case 3. More of these cases are probably surviving the immediate postoperative period as a result of proper blood and plasma replacement. It is some comfort to the surgeon to know that very short lengths of small intestine may be compatible with life. Several instances of survival with only 7 or $8 \mathrm{in}$. of small bowel are recorded (Bothe, Magee, and Driscoll, 1954; Wilkie, 1950; Jackson, Linder, and Berman, 1951; Brezin and Oren, 1954), but the majority with very short lengths exhibit nutritional deficiencies. The subject is thoroughly reviewed by Pullan (1959). Some evidence is accumulating that when a reasonable segment of ileum can be preserved the prognosis, particularly with regard to fat absorption, is improved (Pullan, 1959; Pietz, 1956). Croot (1952) points out that for anatomical reasons distal resections are more common, and few cases are recorded where virtually all the jejunum was resected and a few feet of ileum preserved. Littlefield (1919) records a patient with $4 \mathrm{ft}$. of distal ileum in good health three months later, and McClenahan and Fisher (1950) describe a man who was remarkably well after 18 months with only 1 to $2 \mathrm{ft}$. of ileum and no jejunum. Case 3 in this series is a further example of a patient subjected to a massive resection of proximal small bowel in good health after nine months.

The prevention of recurrence of volvulus offers no problem when a definite cause is found and removed (Cases 4 and 5) or an extensive resection performed (Case 3). In Case 1 a procedure designed to lengthen the root of the mesentery was attempted, but, like Barbosa (1944), I have doubts about its value. In Case 2 the patient's condition precluded all but life-saving measures, but probably such grossly affected bowel becomes sufficiently adherent to prevent recurrence. Recurrent massive volvulus may justify a Noble plication (Noble, 1937).

Post-operatively the possibility of spreading thrombosis occurring in the mesentery must be kept in mind. Deterioration in the condition of a patient, such as in Case 3, is easily overlooked in the immediate post-operative period. It must also be remembered that considerable quantities of protein and fluid may be lost into the bowel wall and into 
the peritoneal cavity after operative relief of an extensive volvulus and generous intravenous replacement of protein and fluid may be needed.

I wish to thank the surgeons at Bristol Royal Infirmary and at Southmead Hospital under whose care these patients were admitted for permission to publish. I am most grateful to Mr. R. V. Cooke for his criticism and encouragement in the preparation of this paper.

\section{REFERENCES}

Aird, I. (1937). Edinb. med. J., 44, 28.

Barbosa, J. J. deCastro. (1944). Amer. J. Surg., 64, 400.
Bothe, F. A. Magee, W. S., and Driscoll, R. H. (1954). Ann. Surg. 140, 755 .

Brezin, D., and Oren, B. G. (1954). Gastroenterology, 26, 895.

Cooke, R. V. (1958). Proc. roy. Soc. Med., 51, 503.

Croot, H. J. (1952). Brit. med. J., 1, 195.

Evans, E. I., and Bigger. I. A. (1947). J. Amer. med. Ass., 133, 513.

Jackson, W. P. U., Linder, G. C., and Berman, S. (1951). S. Afr. J. clin. Sci., 2, 70

Kerr, W. G., and Kirkaldy-Willis, W. H. (1946), Brit. med. J., 1, 799 Littlefield, J. R. (1919). J. Amer. med. Ass., 73, 835.

McCleng, J. (1919). Amer. med. Ass., 73, 835.

McKechnie, R. E., and Priestley, J. T. (1936). Ibid., 34, 286.

Moretz, W. H., and Morton, J. J. (1950). Ann. Surg., 132, 899.

Noble, T. B. (1937). Amer. J. Surg., 35, 41.

Pietz, D. G. (1956). Gastroenterology, 31, 56.

Pullan, J. M. (1959). Proc. roy. Soc. Med., 52, 31.

Scott H. G. (1938). Arch Surg. (Chicago), 36, 816.

Smith, R. (1945). Brit. J. Surg., 32, 510.

Wilkie, W. (1950). S. Afr. med. J., 24,516

Woods, R. S. (1945). Brit. med. J., $2,496$. 